\title{
Aplicación de tecnología LIDAR en El Shincal de Quimivil, Londres, Catamarca
}

\section{Reinaldo A. Moralejo", Diego Gobbo", Daniel Del Cogliano*** y Leandro Pinto*:*}

Recibido: 27 de octubre de 2017

Aceptado: 3 de abril de 2018

\section{Resumen}

El objetivo de este artículo es presentar las potencialidades que posee el uso de la tecnología LIDAR (Light Detection and Ranging) para el estudio de sitios arqueológicos. Para ello daremos a conocer los recientes trabajos realizados en El Shincal de Quimivil e interfluvio de la sierra de Zapata, departamento de Belén, provincia de Catamarca. Se trata de los primeros trabajos con esta tecnología implementados en un sitio arqueológico de la República Argentina. La tecnología LIDAR se basa en la emisión y registro de luz láser reflejada que permite la identificación de distintos niveles de información que el haz encuentra en su camino. Esta propiedad hace posible detectar construcciones a priori ocultas por la capa vegetal. La aplicación de esta metodología en diferentes lugares del mundo ha significado el descubrimiento de estructuras y sitios arqueológicos en los últimos años. Los resultados de este estudio determinaron la presencia de nuevas estructuras arqueológicas no reconocidas hasta el momento y la generación de un Modelo Digital de Terreno (MDT) con una alta resolución espacial.

\section{Application of LIDAR technology at El Shincal de Quimivil, Londres, Catamarca}

\footnotetext{
Abstract

The aim of this article is to assess the potential that LIDAR (Light Detection and Ranging) has for the study of archaeological sites. In doing so, we present our recent research on the site of El Shincal de Quimivil and watershed of the Zapata mountain range, Department of Belén, Province of Catamarca. This is the first research that employs this technology at an archaeological site in the Argentine Republic. LIDAR technology is based on the emission and registration of reflected laser light which thereby allows the identification

* División Arqueología, Facultad de Ciencias Naturales y Museo, Universidad Nacional de La Plata (UNLP) - CONICET. Paseo del Bosque s/n (CP B19ooFWA) La Plata, Buenos Aires, Argentina. E-mail: reinaldomoralejo@yahoo.com.ar; dgobbo@fcnym.unlp.edu.ar

**Departamento de Astrometría, Facultad de Ciencias Astronómicas y Geofísicas, Universidad Nacional de La Plata (UNLP). Paseo del Bosque s/n (CP B19ooFWA) La Plata, Buenos Aires, Argentina. E-mail: ddelco3057@gmail.com

*** Consular. Consultores Argentinos Asociados S. A. Av. Pte. Gral. Julio A. Roca 610, 6 piso (CP C1067ABO)

Ciudad Autónoma de Buenos Aires, Argentina. E-mail: Ipinto@consularsa.com.ar
}

Palabras clave

LIDAR

$3 D$

Modelo Digital de Terreno

Arqueología

El Shincal de Quimivil

Noroeste argentino
Keywords:

LIDAR

3D

Digital Terrain Model Archaeology

El Shincal de Quimivil Northwest Argentina 
of different levels of data that the beam finds across its path. This property makes it possible to detect a priori constructions hidden by layers of vegetation. The use of this methodology in different parts of the world has led to the discovery of previously hidden structures and archaeological sites in recent years. The results of our study revealed the presence of new, previously unregistered, archaeological structures at the site, and the generation of a high-resolution Digital Terrain Model (DTM).

\section{Introducción}

Desde hace más de dos décadas somos testigos de los avances científicos provocados por la incorporación de las tecnologías de la información geográfica. Nuestra disciplina arqueológica no ha quedado afuera de este proceso. Yendo mucho más allá de una utilización pasiva, se ha ocupado de que ciertos aspectos importantes para el estudio del comportamiento humano, como el tiempo y el espacio, se transformen en tópicos esenciales de las nuevas tecnologías. Su uso no implica restituir el paisaje antiguo sino más bien representar mediante modelos la diversa complejidad de la realidad espacial, con el objetivo de poder reflexionar sobre los fenómenos observados.

En el sitio arqueológico de El Shincal de Quimivil, localizado en Londres, departamento de Belén, provincia de Catamarca, se vienen realizando investigaciones arqueológicas desde comienzos del siglo XX. Pero fue recién a partir de 1981, por iniciativa del Dr. Rodolfo Raffino, que comenzaron una serie de investigaciones que hasta la actualidad se mantienen ininterrumpidas. En las mismas han colaborado diversas instituciones como la Universidad Nacional de La Plata (UNLP), el Gobierno de la Provincia de Catamarca, la Dirección Provincial de Antropología de Catamarca, la Municipalidad de Londres, el CONICET, la National Geographic Society, la Agencia Nacional de Promoción Científica y la Fundación Azara.

El Shincal de Quimivil es considerado por algunos investigadores como un Nuevo Cusco, lo que significa que estaría replicando, simbólica y conceptualmente, la capital de todo el Tawantinsuyu (Farrington, 1999; Raffino, Alvis, Baldini, Olivera y Raviña, 1982). También constituyó uno de los principales puntos nodales de la red caminera incaica del Noroeste Argentino. En él confluían el Camino Principal de la Sierra o Qhapaq Ñan que conectaba el valle de Hualfín con la porción más austral de Kollasuyu en Argentina y un camino transversal que conectaba el sitio con enclaves de pastoreo y producción agrícola (Moralejo, 2011). Si bien se han realizado extensos trabajos de investigación y se ha recuperado un registro arqueológico de gran riqueza, las dimensiones del sitio, la diversidad de estructuras y la exuberante vegetación presente hacen que muchos espacios y estructuras permanezcan aún sin ser exploradas. Es por ello que decidimos potenciar la exploración y el estudio arqueológico del sitio y alrededores mediante el uso de una nueva tecnología - no intrusiva - que no registra antecedentes en la arqueología de nuestro país. La misma es conocida con el acrónimo LIDAR que significa Light Detection and Ranging (detección y medición de distancias a partir de luz).

El objetivo de este artículo es presentar las potencialidades que posee el uso de la tecnología LIDAR aéreo para el estudio de sitios arqueológicos. Para ello daremos a conocer los recientes trabajos realizados en El Shincal de Quimivil e interfluvio de la sierra de Zapata. Se trata de los primeros trabajos con esta tecnología implementados en un sitio arqueológico de nuestro país. Los trabajos de campo se realizaron durante el mes de noviembre de 2016 e incluyeron el relevamiento LIDAR y fotográfico aéreo de 5.600 hectáreas en torno del sitio arqueológico y el apoyo con GNSS (Global de Navegación por Satélite) de todas las mediciones. 
Esta técnica permite documentar el paisaje considerando múltiples dimensiones físicas. Se produce un gran volumen de mediciones en poco tiempo, que prometen transformar nuestra comprensión de las sociedades pasadas y los paisajes que manipulan, ayudando así a discusiones sobre los asentamientos a escala de sitio y regional.

Estos estudios son sumamente costosos para cualquier proyecto de investigación que decida llevarlo a cabo, por esta razón, los investigadores de la UNLP impulsamos un trabajo de colaboración científica en el que los diferentes actores aportaron parte de su potencial. Esa iniciativa se coronó a través de un Convenio de Colaboración entre la Universidad Nacional de La Plata, la Universidad Nacional de Catamarca, el Gobierno de Catamarca y la empresa Consular Consultores Argentinos Asociados. La empresa es propietaria de un sistema LIDAR y se ocupó de realizar el vuelo. Las diferentes instancias de procesamiento y el análisis de los resultados fueron abordados por todas las partes mencionadas previamente.

\section{Los inkas y El Shincal de Quimivil}

Los inkas fueron una de las sociedades estatales prehispánicas de mayor alcance territorial en el continente americano. Se trató de una sociedad políticamente expansiva que a lo largo del siglo XV de la Era ejerció su influencia o dominio sobre diversas poblaciones andinas. Esta expansión estuvo basada en diversos intereses sociales, económicos, religiosos y militares que al conjugarse con las realidades locales iban dando forma al paisaje social y cultural en cada región integrante del Tawantinsuyu (Rostworowski de Diez Canseco, 1999). Las estrategias políticas propuestas para estos momentos fueron tanto de carácter coercitivo como diplomáticas, variando de acuerdo al tipo de relaciones establecidas entre los grupos locales y los objetivos estatales. Estos objetivos podrían estar vinculados con la explotación de recursos mineros y agropastoriles y con la conquista simbólica a través de la hospitalidad ceremonial reflejada en diversos elementos del paisaje y la cultura material (Acuto, 1999; Couso et al., 2011; González, 1980; Nielsen y Walker, 1999; Raffino et al., 1978; Williams y D'Altroy, 1998; Williams, Villegas, Gheggi y Chaparro, 2005).

La presencia incaica en el Noroeste Argentino no estuvo ajena a dichos propósitos. Uno de los espacios más emblemáticos en este territorio, ubicado al sur del Kollasuyu, fue El Shincal de Quimivil. Este lugar se encuentra ubicado en la localidad de Londres, departamento de Belén, provincia de Catamarca, sobre el piedemonte del cerro Shincal, entre los ríos Quimivil y Hondo. Ocupa un paisaje muy particular localizado a $1.350 \mathrm{~m}$ s.n.m. y enmarcado en un bosque de algarrobos, chañares, acacias, talas y shinkis. El área de ocupación supera las 30 hectáreas y está compuesta por diversos edificios y/o estructuras que en conjunto conforman un patrón ortogonal que ha sido concebido, planeado y construido de acuerdo al modelo incaico para sus centros políticos regionales (Raffino, 2004). La materialidad presente en el sitio incaico (planeamiento urbano, arquitectura, cerámica, metales, caminos, cuerpos de agua, bloques rocosos) constituye la evidencia actual de una intensa dinámica política que involucraba un amplio territorio que habría sido desde allí controlado. Diferentes sectores del sitio vienen siendo analizados en el marco de diversos trabajos de exploración e investigación desde 1900 (Bruch, 1911; Furque, 1900; González, 1966; Weiser, 1925-1926), pero recién en 1981 un equipo de investigadores dirigido por el Dr. Rodolfo Raffino comienza con un trabajo sistemático de investigación, preservación y divulgación que aún en la actualidad se mantiene gracias a sus discípulos y colaboradores.

Entre los principales componentes de la planta urbana de El Shincal de Quimivil se encuentra una gran plaza amurallada o aukaipata de 175 x $175 \mathrm{~m}$, en cuyo centro se sitúa el ushnu o plataforma ceremonial. Dentro de la misma también se encuentra un 
muro doble de $60 \mathrm{~m}$ de largo con cuatro puertas o vanos trapezoidales que limita la visibilidad desde el acceso principal a la plaza y organiza el espacio en términos de movimiento. Alrededor de la aukaipata se encuentran grandes edificios rectangulares llamados kallanka, donde se realizaban diversas actividades políticas, administrativas y ceremoniales. También se destaca la presencia de varios conjuntos residenciales con un formato regular, conocidos como RPC (Rectángulo Perimetral Compuesto) o kancha en lengua quechua, dispuestas alrededor de la aukaipata y a la vera del camino incaico. Una de ellas, denominada Casa del Curaca estaba destinada a los gobernantes o la élite. Otras servían como residencias permanentes y permitían alojar a los invitados que arribaban a los eventos festivos, algunas eran exclusivas para los habitantes que se encargaban de mantener el sitio a lo largo del año mientras que otras funcionaban como verdaderas kancha-templos. Hacia el oriente y occidente de la plaza se destaca la presencia de dos cerros aterrazados (Cerro Aterrazado Oriental y Cerro Aterrazado Occidental) donde posiblemente se llevaban a cabo prácticas relacionadas con el culto solar (Farrington, 1999; Raffino, 2004; Raffino et al., 1982; Raffino, Iácona, Moralejo, Gobbo y Couso, 2015).

En relación con la actividad administrativa y ceremonial se han encontrado más de cincuenta unidades de almacenamiento o depósitos llamados qollqa dispersos en dos grandes grupos ubicados en lugares con microclima cálido y seco (Capparelli, 1997; Capparelli, Frangi y Kristensen, 2006). También se han hallado varias rocas con estructuras de molienda asociadas con la preparación de comidas y bebidas, así como también varios entierros humanos y canales que abastecían de agua al sitio desde el río Quimivil (Giovannetti, 2009; Salceda y Raffino, 2004).

Otro elemento de importancia es la presencia de un Gnomon o Intihuatana, ubicado hacia el norte de la aukaipata sobre el cerro del Intihuatana, donde se realizaban observaciones solares (Farrington, 1999) y grandes rocas tokankas (rocas wakas sagradas) asociadas al camino incaico (Moralejo, 2011; Moralejo y Aventín Moretti, 2015). En cuanto al Qhapaq Ñan o Camino Principal Andino, este tenía conexión con los enclaves de producción agrícola y pastoreo de Los Colorados y Las Vallas localizados aguas arriba del río Quimivil a través de un camino transversal, y con el tampu Tambillo Nuevo localizado en la ruta por la sierra de Zapata que conducía hacia Chile (Moralejo, 2011; Raffino, Moralejo y Gobbo, 2008).

Este modelo social del paisaje, con una arquitectura monumental estatal y con determinadas regularidades urbanísticas en relación con otros sitios incaicos de los Andes Centrales, condujeron a que El Shincal de Quimivil sea considerado un Nuevo Cusco, tal como se mencionó más arriba. Su importancia ha sido tal en el Noroeste Argentino que de acuerdo a las evidencias materiales arqueológicas e históricas pudo constituir el lugar donde Juan Pérez de Zurita, en 1558, habría fundado Londres de la Nueva Inglaterra (Igareta, 2008) (Figura 1).

\section{¿Por qué es importante realizar un estudio LIDAR en El Shincal de Quimivil? Hipótesis de trabajo}

Todo lo anteriormente mencionado deja en claro que El Shincal de Quimivil constituyó uno de los principales puntos nodales de la red caminera incaica del Noroeste Argentino. Si bien los trabajos de investigación realizados han sido extensos y de gran riqueza en cuanto al registro arqueológico recuperado, los altos niveles de sedimento postdepositacional y la exuberante vegetación, mayormente espinosa, hace que muchos espacios y estructuras permanezcan aún sin ser descubiertos y/o explorados. Existe entonces un amplio espacio de nula visibilidad arqueológica que dificulta cualquier tipo de prospección pedestre y aérea. En este marco y con las hipótesis de que existen, 


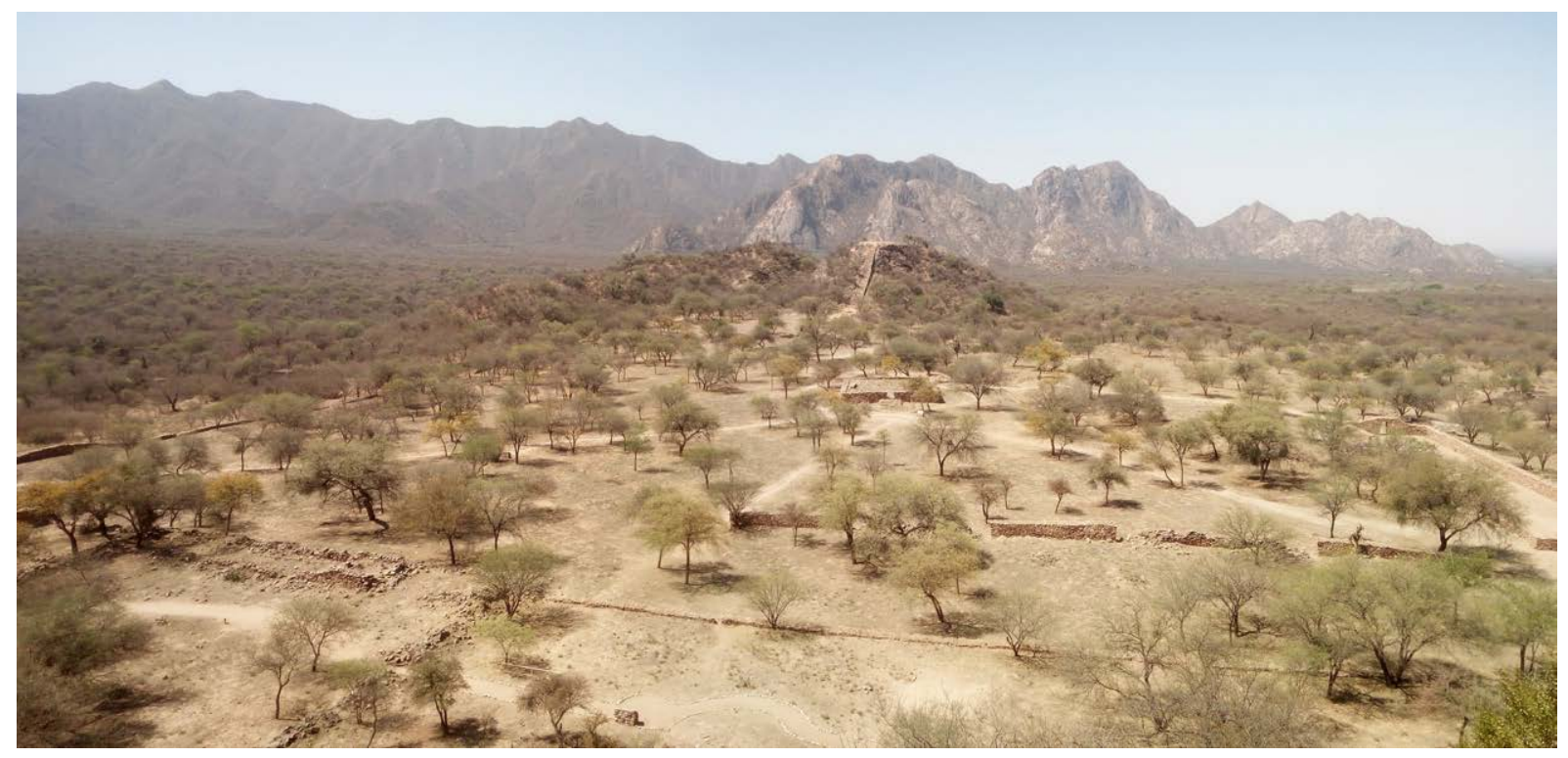

Figura 1. Vista panorámica de El Shincal de Quimivil. En los alrededores del sitio se puede observar la alta densidad de la vegetación que dificulta los estudios arqueológicos.

por un lado, otras estructuras o unidades arqueológicas tipo kancha o rectángulos perimetrales compuestos (RPC) dispuestos alrededor del centro cívico-ceremonial del sitio y, por otro, estructuras o unidades arqueológicas asociadas al camino incaico como parte de una red compleja de apoyo logístico, decidimos emprender un estudio con tecnología LIDAR aérea.

Se trata de una moderna técnica de teledetección que se basa en la medición de las propiedades de los objetos sin que medie contacto con estos. La misma emplea pulsos de láser y constituye una tecnología revolucionaria empleada desde hace unos años para la creación de cartografía de elevaciones, ya que obtiene buenas resoluciones, tanto horizontales como verticales. Esta tecnología resulta óptima para la detección de estructuras cubiertas por vegetación y es capaz, como veremos más adelante, de proporcionar información sobre la posición de la copa de los árboles y de objetos a priori ocultos bajo la vegetación y el suelo, ya que parte de cada haz láser puede reflejarse en las diferentes superficies. El primer resultado de un trabajo con LIDAR es una nube de puntos que, debido a su precisión, permite detectar elementos $(e . g$. arquitectura en superficie) del terreno que con otros sistemas no resultaría posible registrar. Esto hace posible no solo estudiar el terreno sino también obtener otros parámetros tales como la altura de la vegetación, el volumen de la biomasa, etc. Los resultados de su utilización significan un salto cualitativo con respecto a los obtenidos con otras tecnologías (Olaya, 2014).

Otro aspecto importante que motivó este tipo de estudio y que supera lo estrictamente arqueológico, fue la necesidad de obtener un modelo del terreno que nos permitiera conocer en detalle el patrón de distribución de las grandes cárcavas o zanjas que están socavando y destruyendo la superficie del sitio desde hace ya varios años. Los primeros estudios realizados por Niz, Oviedo, Toledo y Lamas (2009) revelan la presencia de grandes surcos o zanjas que pueden alcanzar más de $20 \mathrm{~m}$ de ancho en su cabecera y superar los $7 \mathrm{~m}$ de profundidad. Estas cárcavas se producen por los efectos de las lluvias torrenciales de noviembre a marzo, las cuales generan avenidas de escurrimiento con arrastre de mucho material suelto. Todo ello se ve favorecido por las fuertes insolaciones que agudizan la sequedad del suelo y por la presencia de roedores como los tucu-tucu (género Ctenomys) que generan cuevas donde puede ingresar el agua. Con esta nueva 
1. EI LIDAR terrestre fijo utilizado en El Shincal es propiedad de la Universidad Nacional de La Plata. Con éste se obtuvieron representaciones 3 D de calidad milimétrica y de muy alta densidad de diferentes estructuras habitacionales y ceremoniales del sitio. Para ello se eligió el ushnu o plataforma ceremonial en el centro de la plaza, dos unidades residenciales tipo kancha o RPC y la escalinata de acceso al Cerro Aterrazado Oriental. Esta tarea nace con la intención de obtener un detalle de la arquitectura constructiva de cada una las estructuras en piedra y recrear las diferentes intervenciones realizadas. De esta manera se facilita la virtualización del patrimonio para diversos usos vinculados con la investigación, la conservación y la difusión. información provista por medio de LIDAR se podrá obtener un Modelo Digital del Terreno (MDT) de alta resolución que nos permita observar en detalle la geomorfología del piedemonte donde se instala el sitio. Ello servirá para continuar analizando en profundidad el comportamiento de este rasgo tan erosivo y perjudicial, y así poder generar diferentes políticas de intervención y control en pos de su preservación.

\section{Metodología de levantamiento LIDAR}

El LIDAR es, sin duda, una de las más importantes innovaciones en la recolección e interpretación de datos arqueológicos de los últimos años y está cambiando la naturaleza de la investigación arqueológica (Bewley, Crutchley y Shell, 2005; De Feo, Gobbo y Moralejo, 2013). Esta tecnología permite capturar de manera discreta pero a muy alta resolución y precisión cualquier elemento en tres dimensiones, de tal manera que el producto generado es una nube de puntos de alta densidad en verdadera magnitud, a partir de la cual se pueden realizar cálculos métricos, obtener dibujos, cortes, perfiles o secciones, vectorizar entidades y modelar digitalmente los elementos deseados en 2D/3D en un entorno CAD o SIG. De hecho, esta característica es la principal ventaja de esta tecnología (Arranz Justel, 2013)

Básicamente, el sistema LIDAR es una técnica de teledetección óptica compuesto por un sensor activo, desde el cual se envía un haz muy estrecho de alta energía (láser) hacia el objeto que se desea medir en el que se refleja dicho rayo de manera difusa, siendo esta captada por el sensor que lo recibe y registra. Para determinar la distancia entre el sensor y el objeto que reflejó, mide el tiempo empleado entre la transmisión y la recepción del pulso (Arranz Justel, 2013; Vosselman y Maas, 2010).

Los sistemas LIDAR pueden diferenciarse, según la plataforma donde se monten, en aéreos o terrestres, y en este último caso, a su vez, pueden ser fijos o móviles. En El Shincal de Quimivil, se utilizaron uno aerotransportado y otro fijo terrestre, aunque en el presente artículo solo nos referiremos a las actividades realizadas con el LIDAR aéreo. ${ }^{1}$

El LIDAR aéreo consta de los siguientes elementos: la plataforma o soporte donde se monta el sistema de escáner láser, un Sistema GNSS, una Unidad de Medición Inercial (IMU) y una cámara digital fotogramétrica para realizar fotografías aéreas. Todo lo anterior junto a un sistema informático a bordo que se encarga de la gestión del vuelo, la navegación y el almacenamiento de los datos del sensor (Figura 2).

Estos sistemas registran varios retornos de un único haz emitido y los más modernos almacenan la señal recibida prácticamente en forma continua (a esto se lo conoce como full-waveform). Para el mismo instante en que se emite un pulso láser, el sistema de navegación, basado en GNSS y el IMU, provee las coordenadas y la actitud del sensor. A partir de esa información se pueden determinar las coordenadas espaciales del punto reflejado. El resultado es una nube de puntos con coordenadas espaciales de precisión centimétrica y alta densidad que contiene información de la vegetación, el terreno y eventuales construcciones ocultas debajo de la cobertura vegetal, ya que la señal emitida desde una plataforma aérea por su ubicación espacial respecto al objetivo, en general, permite la identificación de distintos niveles de la información que el haz encuentra en su camino, como las hojas de los árboles, sus ramas y el suelo debajo de ellas.

Un parámetro muy importante en una medición es la densidad de puntos registrados. La misma dependerá en gran medida del tipo de escáner, de la altura de vuelo, de la velocidad del avión sobre el terreno, de las frecuencias de pulsos emitidos y de barrido configurados para cada caso. El procesamiento de los registros LIDAR incluye la solución de una sucesión de líneas de vuelo o pasadas con un porcentaje de 


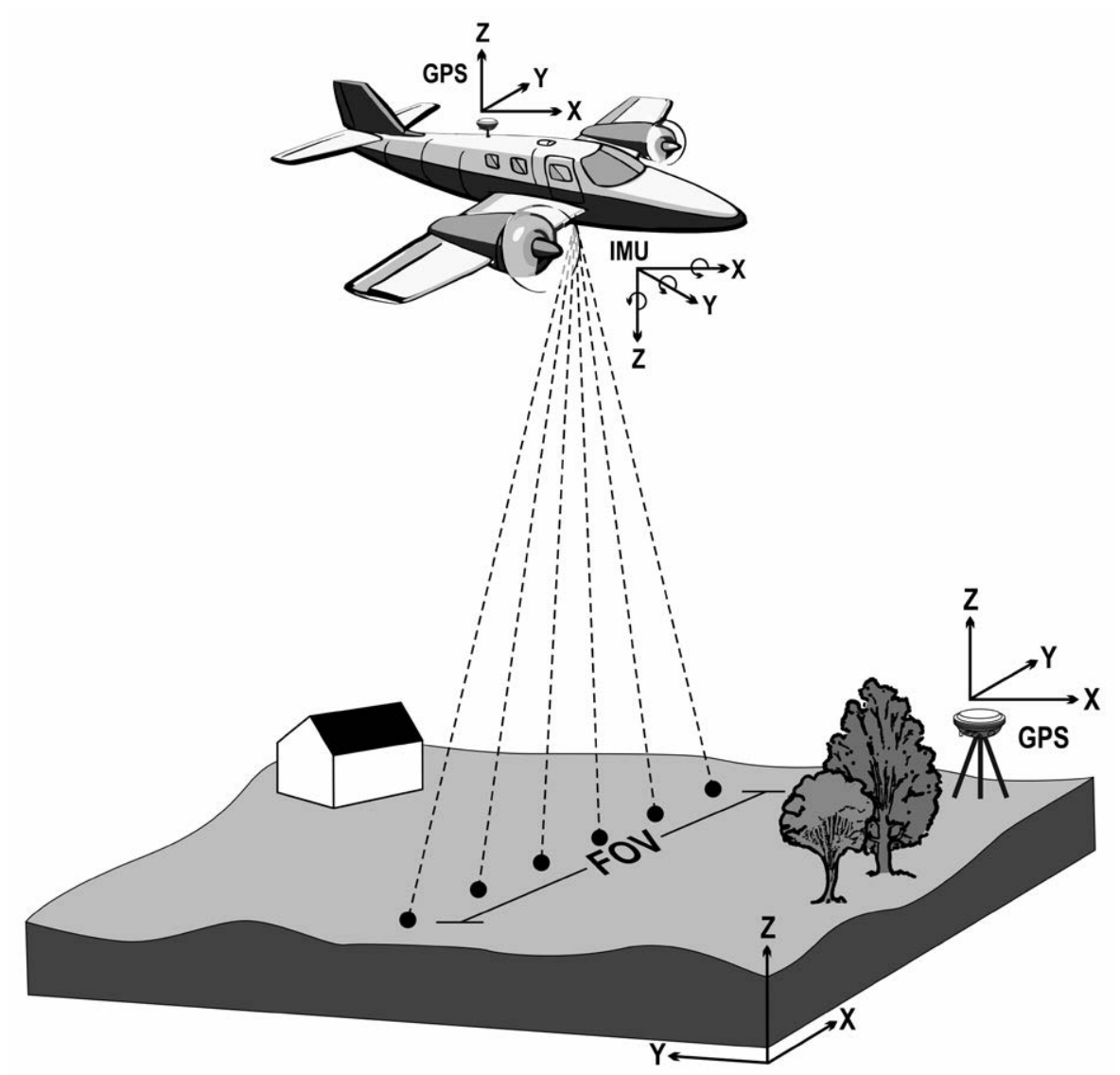

Figura 2. Representación esquemática del estudio LIDAR y sus diferentes componentes (IMU: Unidad de Medición Inercial; FOV: Campo de Visión; GPS: Sistema de Posicionamiento Global).

superposición, que al momento de compararlas presentan diferencias. Esas diferencias son minimizadas durante el procesamiento a través de un ajuste de mínimos cuadrados que incluye transformaciones de similitud afectadas a cada línea de vuelo. En esta instancia la nube de puntos se considera geométricamente resuelta. Empieza entonces una de las fases más importantes en el tratamiento de la información: el proceso de clasificación.

El resultado es una nube de puntos clasificada de acuerdo a los elementos que se pretenden identificar y a la capacidad de los algoritmos aplicados. De hecho, una posible clasificación de los modelos digitales generados vendrá definida por esta selección de puntos. Si el modelo digital está compuesto únicamente por puntos pertenecientes al suelo "desnudo" se hablará de un Modelo Digital del Terreno (MDT). Por el contrario, si el modelo digital incluye además elementos estáticos sobre el suelo, tales como edificios o vegetación, se hablará de un Modelo Digital de Superficies (MDS) (Arranz Justel, 2013). Estos modelos digitales facilitan la ortorectificación de las fotografías aéreas. Esta última consiste en corregir las deformaciones sufridas por la imagen de un objeto o superficie debido a la proyección del sensor, generalmente cónica, y a la diferencia de altura o profundidad de estos.

\section{LIDAR y arqueología}

Es importante destacar que los datos derivados de LIDAR actúan como un documento horizontal y vertical permanente de todo lo que está en el paisaje al momento de recolectar 
los datos (restos arqueológicos, vegetación, topografía), registrando así el estado de la preservación, saqueo, desforestación y construcción moderna en un solo punto en el tiempo (Chase, Chase, Fisher, Leisz y Weishampel, 2012). Bajo esta misma perspectiva será posible elaborar modelos tridimensionales de los recintos o unidades arqueológicas, que permitirán realizar estudios arquitectónicos para comprender sus procesos constructivos y favorecer su preservación (Ching, 1999; Porter, 1983; Sainz, 1990).

La mayor parte de las estructuras arqueológicas se encuentran cubiertas por vegetación de diferentes características, lo que las hace en muchos casos inaccesible a través de una inspección visual o fotográfica. La tecnología LIDAR, sobre todo la basada en el full-waveform, permite un análisis exhaustivo de la cubierta vegetal, ya que se pueden calcular varios parámetros forestales como la extracción de árboles en áreas pequeñas, calcular su altura y diámetro, volumen, diámetro de rama, clasificarlos por especies y otros parámetros como índice de crecimiento o si han sido talados. Asimismo esto hace posible detectar construcciones a priori ocultas por la capa vegetal. La aplicación de esta metodología en diferentes lugares del mundo, y sobre todo en áreas de vegetación frondosa como Belice, Guatemala, Honduras, México o Camboya, permite detectar no sólo construcciones cubiertas por la vegetación, sino también restos de caminos antiguos, terrazas agrícolas y acueductos, entre otros (Chase et al., 2011, 2012; Doneus y Briese, 2006; Doneus, Briese, Fera y Janner, 2008; Golden et al., 2016; Tapete, 2017; von Schwerin, 2016). Así, con relevamientos aéreos de pocos días, es posible obtener resultados que llevarían años con las técnicas convencionales.

La altura de muchas estructuras arqueológicas no superan los $50 \mathrm{~cm}$ y según el sensor y la configuración del vuelo y del terreno, a veces la resolución resultante del producto parecería ser insuficiente. Sin embargo, la existencia de patrones geométricos de las eventuales construcciones colabora en forma determinante para los hallazgos, junto con los registros de intensidad de la señal recibida que varía con la reflectividad de la superficie iluminada por el haz láser. Asimismo, como veremos a continuación, se pueden utilizar una serie de visualizaciones o procesos para facilitar la diferenciación de micro topografías, patrones o estructuras arqueológicas, como el clásico sombreado (Hillshade), sombreado multi-direccional (Multi-Directional Hillshades), mapa de pendientes (Slope Maps), modelo de relieve local (Local Relief Model), factor de visión del cielo (Sky View Factor) y componentes principales de múltiples sombreados (PCA of multiple Hillshades), entre otros (Hutson Scott, 2015; Kokalj, Zakšek y Oštir, 2011, 2013; McCoy, Asner y Graves, 2011; Opitz, 2013; Sithole y Vosselman, 2004; Toumazet, Vautier, Roussel y Dousteyssier, 2017; Zakšek, Oštir y Kokalj, 2011).

\section{Primeros resultados del vuelo LIDAR en El Shincal de Quimivil}

Antes de efectuar el vuelo LIDAR, fue necesario realizar una reunión entre las cuatro partes que conforman el Convenio de Colaboración para discutir con mayor detalle los objetivos y las estrategias de las tareas que se llevarían a cabo. Seguidamente se realizó una presentación formal del Convenio ante la comunidad local, tanto en San Fernando del Valle de Catamarca como en la localidad de Londres. Esta primera instancia incluyó una visita al sitio arqueológico que tuvo como objetivo reconocer las características de las estructuras arqueológicas, tipo de vegetación y terreno que serían posteriormente relevados con tecnología LIDAR.

De esta manera fue posible diseñar las características del vuelo y los parámetros del sensor LIDAR más apropiados para los objetivos planteados. Las mediciones cubrieron no solo el piedemonte de la serranía del Shincal donde yace el sitio homónimo sino también el piedemonte oriental de la sierra de Zapata donde se encuentran los sitios Paraje La Aguada y Tambillos de Zapata, vinculados con El Shincal a través del camino incaico. El vuelo tuvo que contemplar un recorrido entre cerros de diferentes alturas, en tramos 
rectos de unos $30 \mathrm{~km}$, con los márgenes de seguridad adecuados. Por eso, la dirección de las pasadas y la altura del avión tuvieron que ser cuidadosamente programadas.

El relevamiento tuvo lugar el 24 de noviembre de 2016 e incluyó mediciones láser (LIDAR) y fotografía aérea (multiespectral). Se cubrió una superficie total de 5.600 hectáreas en aproximadamente tres horas, con una altura media de $1.000 \mathrm{~m}$ (Figura 3).

El sensor LIDAR utilizado es un Leica ALS 80. El mismo alcanza una frecuencia de barrido de $200 \mathrm{hz}$ (200 líneas por segundo) y emite pulsos láser a una frecuencia máxima de $1 \mathrm{Mhz}$. La cámara fotogramétrica RCD30 es multiespectral y tiene la capacidad de registrar la región del infrarrojo correspondiente al infrarrojo cercano (780 - 880 nanómetros) con una resolución de 80 megapíxeles. Todo el sistema pesa aproximadamente $100 \mathrm{~kg}$ y fue montado en un avión Aerocomander 500S.

Una red de estaciones GNSS operando sobre puntos POSGAR07 sirvieron de apoyo al sistema de navegación aéreo, constituido por un receptor GNSS geodésico y la Unidad de Medición Inercial (IMU). Las estaciones GNSS de tierra se distribuyeron de tal manera que durante la inicialización del IMU la distancia al avión sea menor a los 10 $\mathrm{km}$ y posteriormente no supere los $50 \mathrm{~km}$.

La solución diferencial de la trayectoria utilizó los datos GNSS en combinación con la información del IMU (aceleraciones y ángulos Roll-Pitch-Yaw), produciendo para cada instante la posición y la orientación del sensor. Las distancias y ángulos registrados para cada pulso láser y sus diferentes retornos, fueron procesados a continuación con la trayectoria calculada para generar las coordenadas espaciales de cada punto reflejado en el marco de referencia POSGAR07.

El resultado fue una nube de puntos 3D con coordenadas espaciales de calidad centimétrica ( $10 \mathrm{~cm}$ en la vertical y de hasta $14 \mathrm{~cm}$ en la horizontal) y alta densidad $\left(18 \mathrm{pts} / \mathrm{m}^{2}\right)$ que contiene información de la vegetación alta, media y baja, el terreno y las eventuales construcciones ocultas debajo de la cobertura vegetal. Esta medición fue diseñada a partir del reconocimiento del terreno realizado anteriormente a la ejecución del vuelo, de modo que permitiera disponer de suficiente cantidad de retornos desde la superficie del suelo y desde las construcciones que se encontraban ocultas por aquella.

Para el modelado del terreno fue suficiente disponer de uno o dos puntos por metro cuadrado dependiendo del tipo de terreno, mientras que para la detección de pircados ocultos de 20 a $50 \mathrm{~cm}$ de altura por $60 \mathrm{~cm}$ de ancho, como en el caso de El Shincal, la densidad requerida fue bastante mayor. Por eso se configuró la cantidad de pulsos por segundo (PR: Pulse Rate), el FOV, la frecuencia de escaneo, la altura del vuelo y la superposición entre líneas de vuelo adyacentes de manera tal de satisfacer estos requerimientos básicos.

Cuando la solución de todos los puntos fue integrada, se notaron desplazamientos entre las nubes de puntos correspondientes a diferentes líneas de vuelo. Se inició entonces un proceso de ajuste entre las diferentes pasadas para minimizar las diferencias entre ellas. Para eso se clasificó la nube de puntos, distinguiendo los puntos correspondientes al terreno que debe coincidir para pasadas adyacentes en los sectores de superposición. Se utilizó el método de mínimos cuadrados y se determinaron los parámetros de transformación de similitud para todas las trayectorias. Estas transformaciones se aplicaron a toda la nube, aunque se calcularon solo a partir de los puntos del suelo desnudo o ground. Se obtuvo así la nube matemáticamente ajustada con un ruido típico de $5 \mathrm{~cm}$. Finalmente, gracias a los puntos de control en tierra obtenidos a partir de mediciones cinemáticas GNSS, se vinculó la nube ajustada al marco de referencia geodésico POSGAR07. 


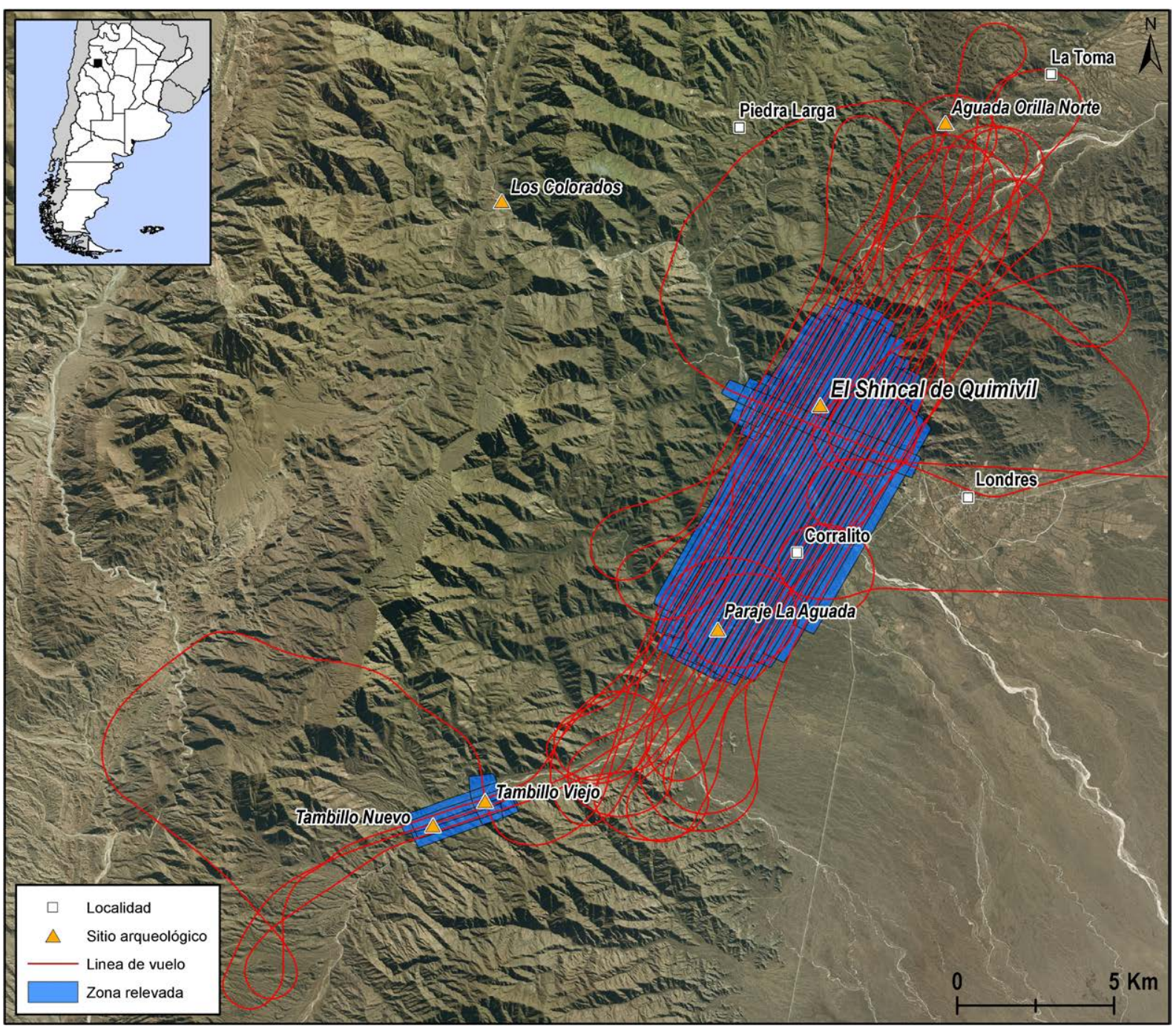

Figura 3. Plan del vuelo realizado.

\section{Análisis de estructuras desconocidas}

Una vez que la nube estuvo completa y ajustada, se inició el proceso de filtrado y clasificación que permitió identificar la información perteneciente a la vegetación, al terreno y las eventuales construcciones. Los algoritmos estándares no permitieron detectar las estructuras bajas ocultas por la vegetación, por lo que fue necesario diseñar estrategias novedosas que aíslen suficientes puntos característicos de los elementos de interés. Cuando los mismos son asociados a patrones geométricos, se convierten en potencial información de interés arqueológico. No obstante, es conveniente desarrollar algoritmos y filtros orientados para uso arqueológico (Figura 4).

Una vez realizada la clasificación, se utilizaron diferentes procesos de visualización que permitieron acentuar los rasgos débiles o micro, así como también identificar patrones estructurales arquitectónicos. Para ello utilizamos el software libre Relief Visualization Toolbox (Kokalj et al., 2011, 2013; Zakšek et al., 2011). Algunos de los procesos más eficientes fueron el factor de visión del cielo (Sky View Factor) y los componentes principales de múltiples sombreados (PCA of multiple Hillshades) (Figura 5). 

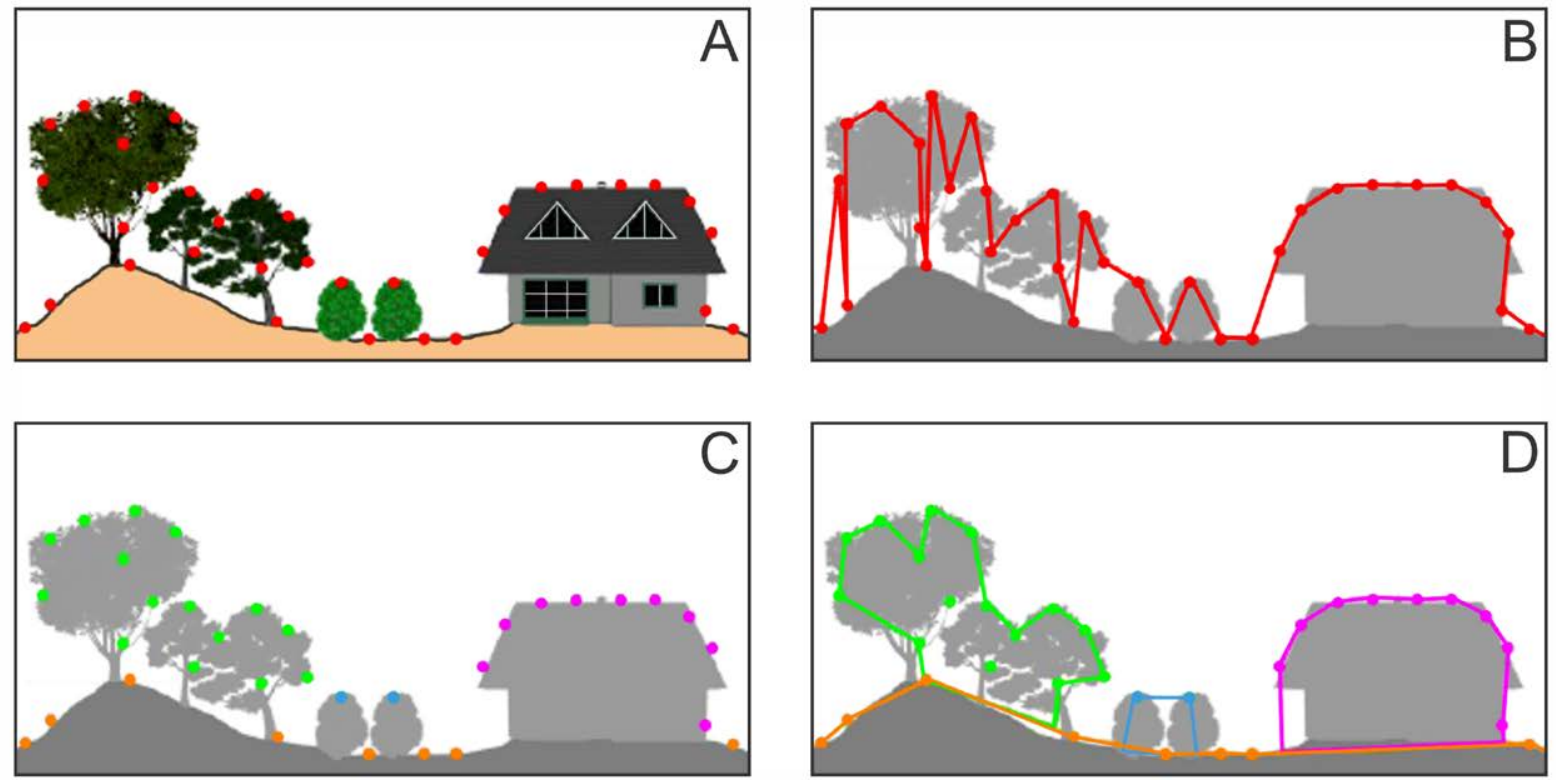

Figura 4. A) Puntos mostrados de manera independiente; B) Línea de puntos escaneada; C) Puntos diferenciados por color en función del objeto al que pertenecen; D) Entidades generadas después de la clasificación de los puntos: en verde se indica la vegetación alta, en violeta las edificaciones, en azul la vegetación media y en naranja el suelo desnudo o ground. (Imagen tomada y modificada de Arranz Justel, 2013, p. 193).

Por último una vez realizado el MDT, se georreferenciaron y ortorrectificaron las imágenes tomadas por la cámara fotogramétrica, generando un mosaico fotográfico de todo lo relevado con una resolución espacial de aproximadamente $5 \mathrm{~cm}$.

Toda esta tarea de gabinete fue llevada a cabo de manera transdisciplinaria entre especialistas de la empresa Consular Consultores Argentinos Asociados e investigadores de la UNLP. Posteriormente, con la necesidad de determinar la sensibilidad del método y ajustar los diferentes filtrados y algoritmos, fue importante corroborar in situ toda la información generada en gabinete.

La primera etapa de contrastación de los datos generados en gabinete con respecto a las observaciones de terreno se realizó sobre un radio de tres kilómetros en torno del sitio El Shincal en marzo de 2017. Para ello contamos con el fotomosaico mencionado y las coordenadas geográficas de cada punto y/o zonas de interés. La tarea involucró dos equipos de investigadores que debieron internarse en campos de vegetación densa de difícil acceso. Se exploraron un total de 39 zonas o puntos correspondientes a hechos existentes, lo que constituye una validación muy importante de la metodología utilizada. El 26\% de los mismos resultaron ser estructuras arqueológicas y el resto rasgos sobresalientes del terreno, algunos de ellos producidos naturalmente (e.g. bloques de piedra acumulados por drenaje natural, montículos de tierra, árboles y restos de madera de cardón derrumbados) y otros por acciones humanas recientes (e.g. albardones de tierra para control hídrico) (Figuras 6 y 7). En cuanto a las nuevas estructuras halladas, éstas corresponden a muros dobles de hasta $60 \mathrm{~cm}$ de ancho cubiertos de vegetación, algunas de las cuales se encontraban localizadas muy próximas a El Shincal. Las mismas serán prontamente incorporadas al plano y se presentarán en otro artículo acerca de una reevaluación del espacio arquitectónico del sitio. Por otro lado, también se pudo relevar con precisión el camino incaico que recorre el sitio de noreste a suroeste (Figura 8).

En el caso de las estructuras que se encuentran parcialmente cubiertas de vegetación, los diversos métodos de visualización de la nube de puntos demostraron tener una correspondencia muy clara con lo observado en el terreno (Figura 9). 

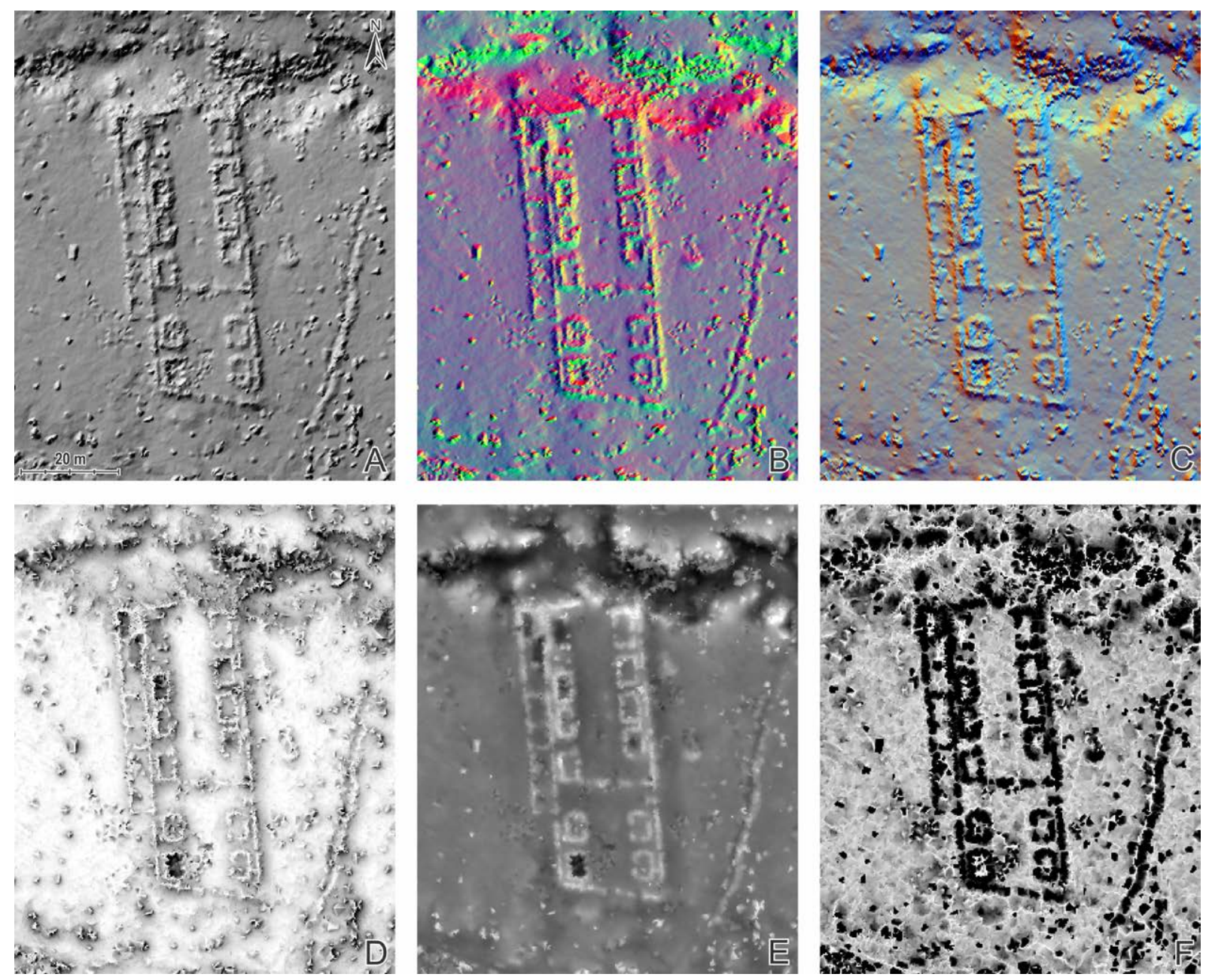

Figura 5. Diferentes modelos de visualización generados con el software Relief Visualization Toolbox. A) Analytical hillshading; B) PCA of multiple hillshadings; C) Hillshadings from multiple directions RGB; D) Anisotropic sky-view factor; E) Simple local relief model; F) Openness negative. Los diferentes cuadros comparten la misma escala.

\section{Consideraciones finales}

Las actividades con LIDAR aéreo descriptas en este trabajo representan el primer caso de estudio científico en nuestro país donde se utiliza este tipo de tecnología láser 3D. Para su realización fue determinante la colaboración de los ámbitos público y privado, expresada a través de la participación de las Universidades Nacionales de Catamarca (UNCa) y La Plata (UNLP), la Secretaría de Estado de Cultura de Catamarca y la empresa Consular Consultores Argentinos Asociados. Esto demandó la elaboración de un Convenio de Colaboración donde se indicaron los objetivos generales y específicos del proyecto, los compromisos asumidos por cada una de las partes y un cronograma de actividades, el cual tuvo su correlato en la práctica.

Gracias a este trabajo se puede potenciar la exploración arqueológica de El Shincal de Quimivil y la ruta de la sierra de Zapata, que lo conecta con la porción más austral del Kollasuyu, mediante el uso de nuevas tecnologías no intrusivas. Asimismo, su concreción abre un amplio abanico de investigaciones que excede a los sitios relevados y lo estrictamente arqueológico. Es decir, se ha generado nueva información de interés para diferentes usuarios tanto del ámbito nacional como internacional, pero muy especialmente para el gobierno de la provincia de Catamarca y la Municipalidad de 

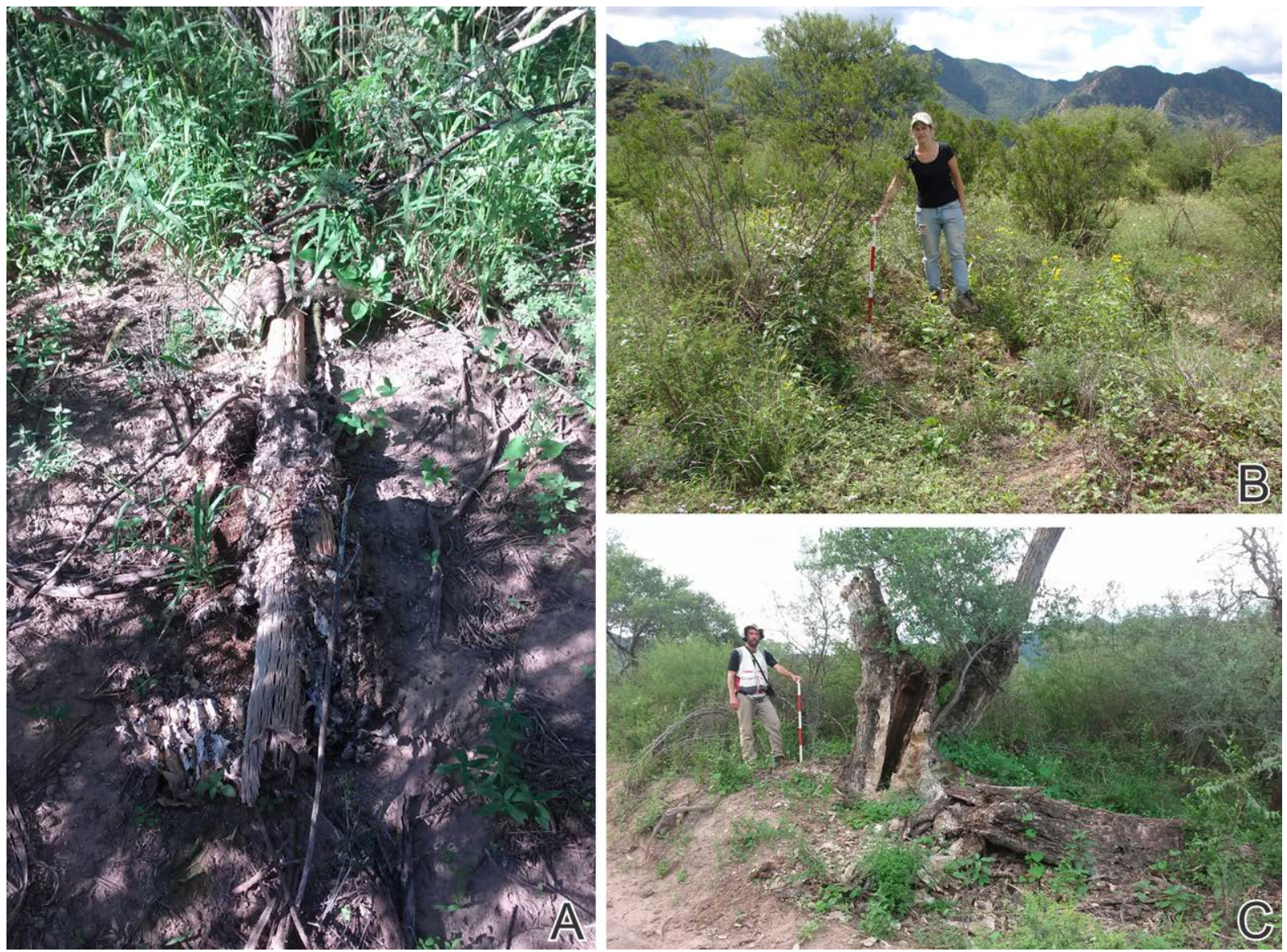

Figura 6. Rasgos de origen natural detectados. A) Restos de un cardón derrumbado, el cual posee un ancho de $30 \mathrm{~cm}$ y un largo de 2,6 m; B) Montículo de tierra; C) Restos de un árbol caído.
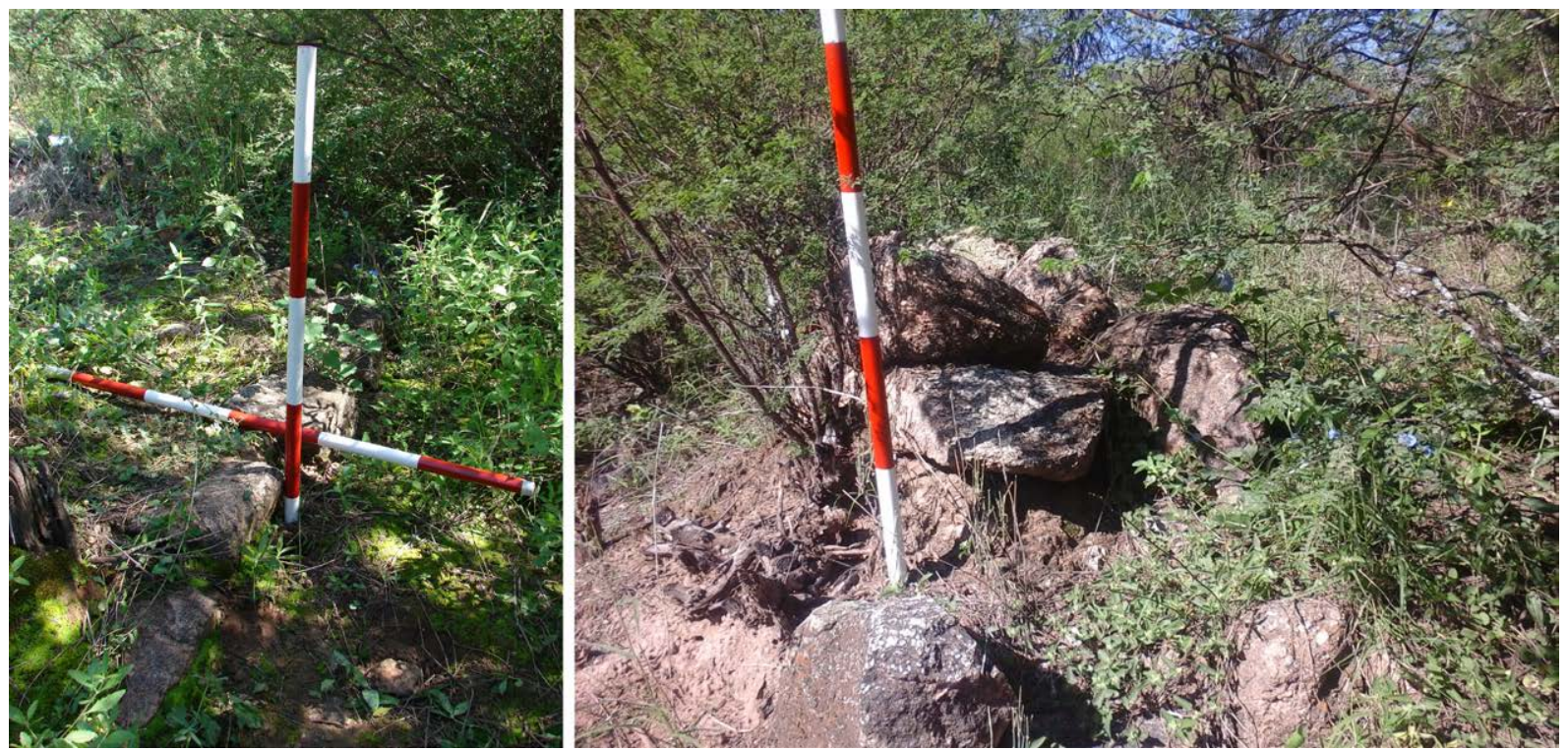

Figura 7. Rasgos arqueológicos detectados. 

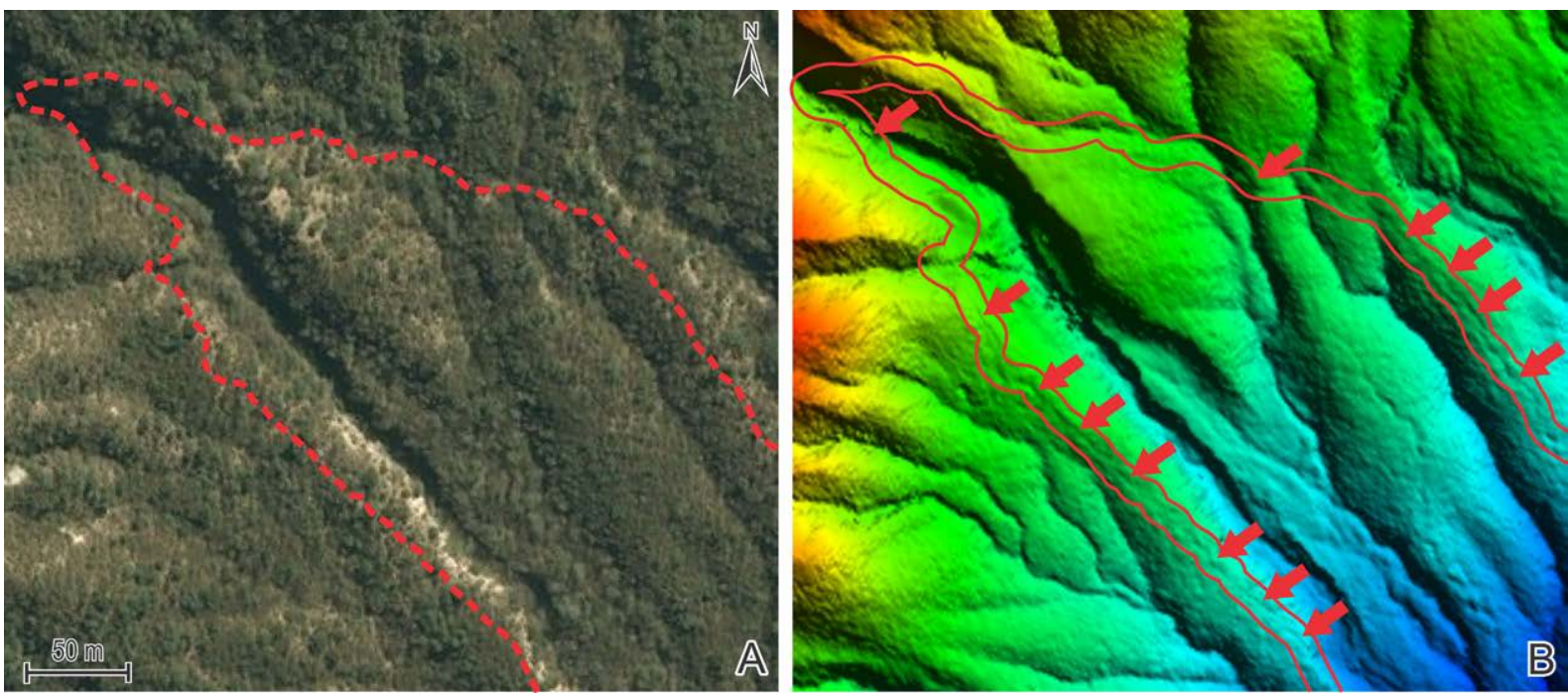

Figura 8. Tramo de Qhapaq Ñan. A) Debajo de la vegetación; B) Fácilmente reconocible con el MDT (Modelo Digital de Terreno).

2. Como sería el caso de la integración con las nubes de puntos producidas con el LIDAR terrestre fijo de la UNLP.
Londres. El Modelo Digital del Terreno permitirá a los responsables del gobierno encarar una solución hidráulica que detenga el avance de las cárcavas y así evitar la progresiva destrucción de amplios sectores del sitio. Cabe resaltar que durante los días de trabajo también se brindaron charlas informativas a los colegios de la zona y grupos de personas que visitaban el sitio. Ello es muy importante ya que colabora con la difusión de todas las investigaciones llevadas a cabo en el lugar.

La magnitud de los relevamientos planteados también representa un hecho significativo, ya que se dispone de una densa nube de puntos, georreferenciada e integrable con otra información georreferenciada, sobre unas 5.600 hectáreas. ${ }^{2}$

De acuerdo con los objetivos de este artículo y considerando las hipótesis planteadas al comienzo de nuestros trabajos con LIDAR aéreo, el hallazgo de nuevas estructuras debajo de la vegetación (todas ocultas a priori), hora debidamente georreferenciadas en el marco geodésico POSGAR07, permite generar nuevas investigaciones vinculadas con el diseño ortogonal de los sitios, la movilidad a través del paisaje, la logística de comunicación proyectada por los inkas y la relación entre las estructuras arquitectónicas y la evolución del paisaje.

La sensibilidad de la metodología demostró una resolución de $10 \mathrm{~cm}$ en altura (aún sobre montículos) y de hasta $14 \mathrm{~cm}$ en la horizontal. De este modo, el relevamiento de El Shincal y zonas aledañas, donde se trata de detectar nuevas construcciones en piedra de 20 a 50 $\mathrm{cm}$ de altura por $60 \mathrm{~cm}$ de ancho, constituye un caso con características diferentes al de otras aplicaciones equivalentes en el mundo, que involucran construcciones medianas o grandes. De esta manera, se pueden investigar nuevos sectores (aún inexplorados) con la posibilidad de detectar vestigios de caminos, terrazas agrícolas, acueductos, depósitos de almacenamiento y estructuras residenciales y ceremoniales sin la necesidad de tener que adentrarnos a ciegas entre la tupida vegetación. Por otro lado, el hallazgo de rasgos no arqueológicos sobresalientes del terreno, ya sean de origen antrópico o natural, también señala la alta sensibilidad de la técnica lograda durante el estudio.

Por ejemplo, existen trabajos donde se busca, a través de diferentes relevamientos LIDAR, detecciones automáticas de restos arqueológicos en base a las características morfométricas de estructuras ya conocidas, y recientemente se han desarrollado métodos más sofisticados con algoritmos más complejos que buscan una detección 

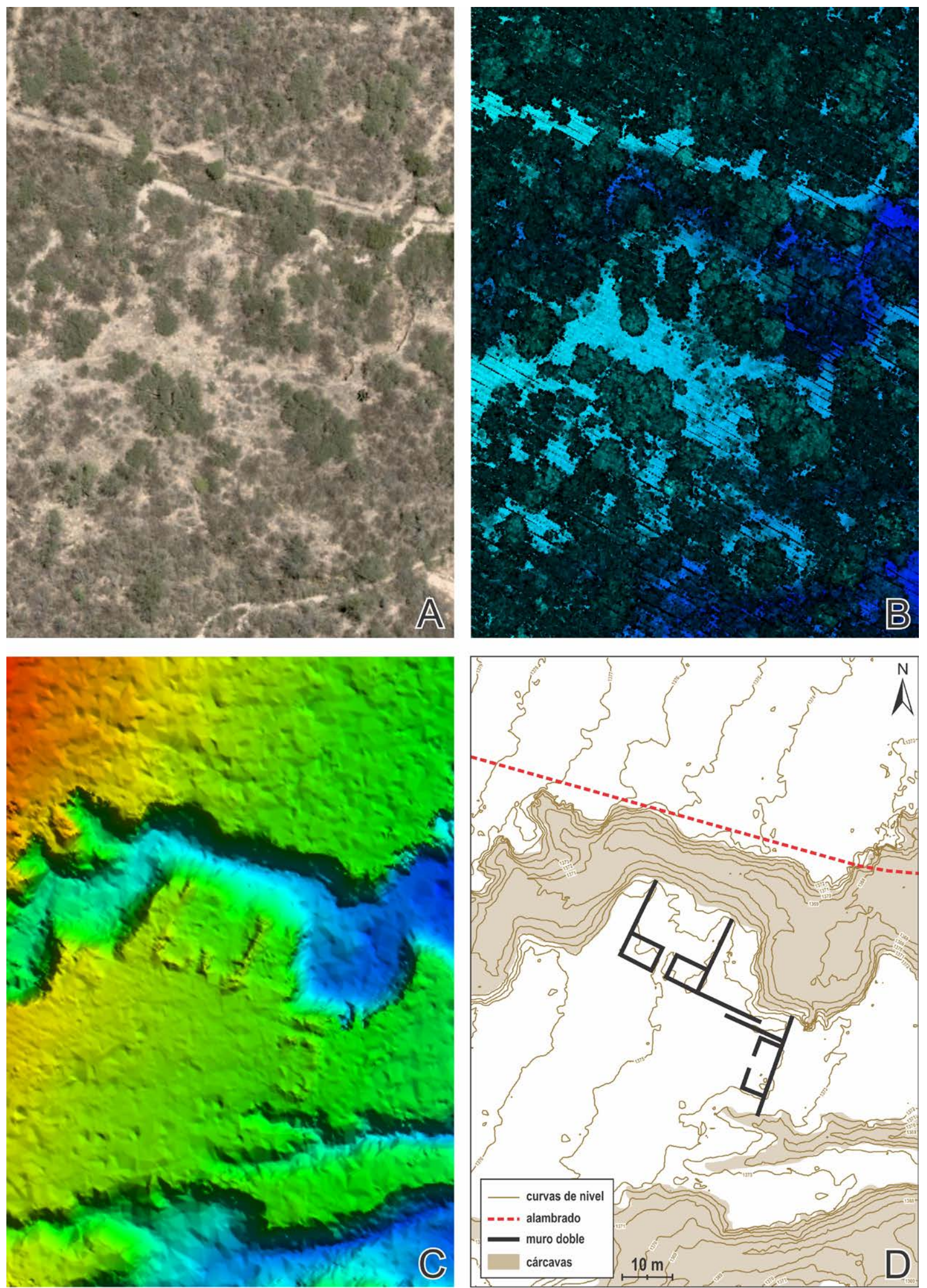

Figura 9. Vista de una de las unidades residenciales o kancha de El Shincal de Quimivil. A) Fotografía aérea; B) Nube de puntos LIDAR; C) Modelo Digital de Superficie sin la cobertura vegetal; D) Modelo plani-altimétrico de la kancha. 
automática de restos arqueológicos (Toumazet et al., 2017; Traviglia, Cowley y Lambers, 2016). Consideramos entonces que será importante continuar con la optimización de las estrategias de medición, procesamiento, filtrado, clasificación y análisis de la información LIDAR en pos de favorecer el conocimiento actual. Para el caso de las nuevas estructuras halladas será importante realizar tareas de prospección y excavación con el propósito de comprender el rol que cumplían dentro del paisaje.

La aplicación de esta técnica no tradicional al estudio de un conjunto de sitios arqueológicos pone en evidencia el aporte a la arqueología de disciplinas como la geodesia, cuando se hace uso de las nuevas tecnologías. Esta actividad interdisciplinaria aporta un gran nivel de integración de información de diferentes características que apuesta a formas avanzadas de virtualización que generan nuevas perspectivas de trabajo en pos de la investigación, preservación y difusión del patrimonio cultural y natural de El Shincal de Quimivil. En conjunto, todo lo mencionado será importante para enriquecer el conocimiento de las problemáticas sociales del horizonte incaico en el Noreste Argentino y particularmente en la provincia de Catamarca. Asimismo, los trabajos realizados en conjunto con la comunidad de Londres, vinculados con la revalorización arqueológica y patrimonial del sitio El Shincal contribuirán a constituir un posicionamiento identitario en cuanto al pasado y presente de la región, tal como lo vienen haciendo hace más de treinta años.

\section{Agradecimientos}

Este trabajo se enmarca dentro de las tareas que venimos desarrollando en el proyecto "La construcción del paisaje vial en la Sierra de Zapata y sur del Valle de Hualfín: prácticas sociales en El Shincal de Quimivil, Tambillos de Zapata, Los Colorados y Las Vallas" dirigido por el Dr. Reinaldo A. Moralejo. Queremos agradecer a la Municipalidad y comunidad de Londres por permitirnos realizar este estudio. A la Encargada de El Sitio El Shincal de Quimivil Lic. Paula Espósito, junto a su equipo de guías turísticos y colaboradores, por el constante apoyo durante la gestión y realización del relevamiento. A la Secretaría de Estado de Cultura y Dirección Provincial de Antropología de Catamarca por brindarnos los permisos correspondientes. A la empresa Consular Consultores Argentinos Asociados por la realización del vuelo. A la Agrimensora Mirtha Rodríguez Zar y su equipo de colaboradores de la Facultad de Ciencias Tecnológicas y Aplicadas de la UNCa por su colaboración durante las tareas de medición con GNSS. A los Licenciados Daniel Muntz, Lautaro Simontacchi, Víctor Mezio, Guillermina Couso por su participación en las tareas de campo. Al Dr. César Parcero-Oubiña y al Instituto de Ciencias del Patrimonio de Santiago de Compostela (INCIPIT-CSIC) por recibir y coordinar la pasantía de investigación del Dr. Reinaldo A. Moralejo. A la Universidad Nacional de La Plata y al CONICET por el financiamiento de las investigaciones en el lugar. Por último, también agradecemos a los evaluadores que contribuyeron a mejorar el manuscrito con sus sugerencias. 


\section{Referencias citadas}

" Acuto, F. A. (1999). Paisaje y dominación: la constitución del espacio social en el Imperio Inka. En A. Zarankin y F. A. Acuto (Eds.), Sed Non Satiata. Teoría Social en la Arqueología Latinoamericana Contemporánea (pp. 33-75). Buenos Aires: Ediciones del Tridente.

》 Arranz Justel, J. J. (2013). Diseño, optimización y análisis de sistemas basados en técnicas láser, para el modelado geométrico, registro y documentación, aplicados a entidades de interés patrimonial. (Tesis Doctoral inédita), Universidad Politécnica de Madrid, España.

" Bewley, R., Crutchley, S. y Shell, C. (2005). New light on an ancient landscape: Lidar survey in the Stonehenge World Heritage Site. Antiquity, 79(305), 636-647.

» Bruch, C. (1911). Exploraciones Arqueológicas en las Provincias de Tucumán y Catamarca. Buenos Aires: Coni Hermanos.

"Capparelli, A. (1997). Reconstrucción ambiental de la instalación arqueológica Inka de El Shincal. (Tesis Doctoral inédita), Universidad Nacional de La Plata, Argentina.

》 Capparelli, A., Frangi, J. L. y Kristensen, M. J. (2006). El urbanismo Inka y su vinculación con mesoclimas en el sitio "El Shincal de Quimivil" (Provincia de Catamarca, Argentina). Intersecciones en Antropología, 7, 163-177.

"Chase, A. F., Chase, D. Z., Fisher, C., Leisz, S. y Weishampel, J. (2012). Geospatial revolution and remote sensing LiDAR in Mesoamerican archaeology. Proceedings of the National Academy of Sciences, 109(32), 12916-12921.

" Chase, A. F., Chase, D. Z., Weishampel, J. F., Drake, J. B., Shrestha, R. L., Slatton, K. C., Awe, J. y Carter, W. E. (2011). Airborne LiDAR, archaeology, and the ancient Maya landscape at Caracol, Belize. Journal of Archaeological Science, 38, 387-398.

» Ching, F. (1999). Manual de dibujo arquitectónico. Barcelona: G. Gili.

"Couso, M. G., Moralejo, R. A., Giovannetti, M. A., del Papa, L. M., Páez, M. C., Gianelli, J., Giambelluca, L. R., Arnosio, M. y Raffino, R. A. (2011). Análisis de la variabilidad material del Recinto 1 - Kancha II: aportes para una comprensión de la política incaica en El Shincal de Quimivil. Arqueología, 17, 35-55.

" De Feo, M. E., Gobbo, D. y Moralejo R. A. (2013). Hacer arqueología desde las alturas. Revista Museo, 26, 23-30.

》Doneus, M. y Briese, C. (2006). Digital terrain modeling for archaeological interpretation within forested areas using full-waveform laser scanning. En M. loannides, D. Arnold, F. Niccolucci y K. Mania (Eds.), Proceedings of the 7 th International Symposium on Virtual Reality, Archaeology and Cultural Heritage (pp. 155-162). Aire-la-Ville: Eurographics Association.

»Doneus, M., Briese, C., Fera, M. y Janner, M. (2008). Archaeological prospection of forested areas using full-waveform airborne laser scanning. Journal of Archaeological Science, 35(4), 882-893.

» Farrington, I. S. (1999). El Shincal: un Cusco del Kollasuyu. En C. Diez Marín (Ed.), Actas del XII Congreso Nacional de Arqueología Argentina (Tomo I) (pp. 53-62). La Plata: Facultad de Ciencias Naturales y Museo, Universidad Nacional de La Plata.

"Furque, H. (1900). Las ruinas de Londres de Quinmivil (Catamarca). Anales de la Sociedad Científica Argentina, L, 166-171. 
"Giovannetti, M. (2009). Articulación entre el sistema agrícola, sistema de irrigación y áreas de molienda como medida del grado de ocupación Inka en El Shincal y Los Colorados (Valle de Hualfín, Provincia de Catamarca). (Tesis Doctoral inédita), Universidad Nacional de La Plata, Argentina.

" Golden, Ch., Murtha, T., Cook, B., Shaffer, D. S., Schroder, W., Hermitt, E. J., Alcover Firpi, O. y Scherer, A. K. (2016). Reanalyzing environmental lidar data for archaeology: Mesoamerican applications and implications. Journal of Archaeological Science: Reports, 9, 293-308.

" González, A. R. (1966). Las ruinas del Shincal. En Actas del Primer Congreso de Historia de Catamarca (Tomo III) (pp. 15-28).San Fernando del Valle de Catamarca: Junta de Estudios Históricos de Catamarca.

»González, A. R. (1980). Patrones de asentamiento incaico en una provincia marginal del imperio; implicancias socio-culturales. Relaciones de la Sociedad Argentina de Antropología, XIV(1), 63-82.

" Hutson Scott, R. (2015). Adapting LiDAR data for regional variation in the tropics: A case study from the Northern Maya Lowlands. Journal of Archaeological Science: Reports, 4, 252-263.

" Igareta, A. (2008). Últimas noticias desde Londres. El sitio histórico más antiguo de la arqueología de Catamarca. (Tesis Doctoral inédita), Universidad Nacional de La Plata, Argentina.

» Kokalj, Ž., Zakšek, K. y Oštir, K. (2011). Application of sky-view factor for the visualization of historic landscape features in LiDAR-derived relief models. Antiquity, 85, 263-273

» Kokalj, Ž., Zakšek K. y Oštir K. (2013). Visualizations of Lidar Derived Relief Models. En R. Opitz y C. David Cowley (Eds.), Interpreting Archaeological Topography: 3D Data, Visualisation and Observation (pp. 100-114). Oxford: Oxbow Books.

» McCoy, M. D., Asner, G. P. y Graves, M. W. (2011). Airborne LiDAR survey of irrigated agricultural landscapes: an application of the slope contrast method. Journal of Archaeological Science, 38, 2141-2154.

"Moralejo, R. A. (2011). Los Inkas al sur del Valle de Hualfín: organización del espacio desde una perspectiva paisajistica. (Tesis Doctoral inédita), Universidad Nacional de La Plata, Argentina.

» Moralejo, R. A. y Aventín Moretti, M. (2015). Los estudios de vialidad en El Shincal de Quimivil. En R. A. Raffino, L. A. lácona, R. A. Moralejo, D. Gobbo y M. G. Couso (Comps.), Una Capital Inka al Sur del Kollasuyu: El Shincal de Quimivil (pp. 105-125). Buenos Aires: Fundación de Historia Natural Félix de Azara.

» Nielsen, A. E. y Walker, W. H. (1999). Conquista ritual y dominación política en el Tawantinsuyu: el caso de Los Amarillos (Jujuy, Argentina). En A. Zarankin y F. A. Acuto (Eds.), Sed Non Satiata. Teoría Social en la Arqueología Latinoamericana Contemporánea (pp. 153-169). Buenos Aires: Ediciones del Tridente.

» Niz, A. E., Oviedo, J. A., Toledo, J. C. y Lamas, C. (2009). Aportes de la Geología e Ingeniería a la recuperación de áreas afectadas por carcavamiento. En Investigaciones en Facultades de Ingeniería del NOA, V Jornadas de Ciencia y Tecnología de las Facultades de Ingeniería del NOA (Tomo 1) (pp. 12-17). Salta: Editorial de la Universidad Nacional de Salta, Facultad de Ingeniería.

» Olaya, V. (2014). Sistemas de Información Geográfica, http://volaya.github.io/libro-sig/ (Acceso: 4 de Septiembre, 2017).

»Opitz, R. (2013). ALS and Archaeology. CAST Technical Publications Series. 1196o. http:// gmv.cast.uark.edu/scanning/airborne-laser-scanning/als-and-archaeology (Acceso: 21 de Mayo, 2017) 
» Porter, T. (1983). Manual de técnicas gráficas para arquitectos, diseñadores y artistas. Barcelona: G. Gili.

» Raffino, R. A. (Comp.) (2004). El Shincal de Quimivil. San Fernando del Valle de Catamarca: Editorial Sarquis.

» Raffino, R. A., Albornoz, A. M., Bucci, A. L., Crowder, R., lácona, A., Olivera, D. E. y Raviña, G. (1978). La ocupación Inka en el N.O. Argentino: actualización y perspectivas. Relaciones de la Sociedad Argentina de Antropología, XII(NS), 95-121.

» Raffino, R. A., Alvis, R. J., Baldini, L. N., Olivera, D. E. y Raviña, M. G. (1982). HualfínEl Shincal-Watungasta. Tres casos de urbanización Inka en el N.O. argentino. En Actas del IX Congreso Nacional de Arqueología (pp. 470-497). La Serena: Sociedad Chilena de Arqueología, Museo Arqueológico de La Serena.

» Raffino, R. A., Moralejo, R. A. y Gobbo, D. (2008). El dominio Inka en la Sierra de Zapata (NOA). Investigaciones y Ensayos, 56, 309-332.

»Raffino, R. A., lácona, L. A., Moralejo, R. A., Gobbo, D. y Couso, M. G. (Comps.) (2015). Una Capital Inka al Sur del Kollasuyu: El Shincal de Quimivil. Buenos Aires: Fundación de Historia Natural Félix de Azara.

» Rostworowski de Diez Canseco, M. (1999). Historia del Tahuantinsuyu. Lima: Instituto de Estudios Peruanos.

»Sainz, J. (1990). El dibujo de arquitectura; teoría e historia de un lenguaje gráfico. Madrid: Nerea.

»Salceda, S. y Raffino, R. A. (2004). El Hombre de El Shincal. En R. A. Raffino (Comp.). El Shincal de Quimivil (pp. 165-178). San Fernando del Valle de Catamarca: Editorial Sarquis.

»Sithole, G. y Vosselman, G. (2004). Experimental comparison of filter algorithms for bareEarth extraction from airborne laser scanning point clouds. Journal of Photogrammetry $\varepsilon$ Remote Sensing, 59, 85-101.

» Tapete, D., Banks, V., Jones, L., Kirkham, M. y Garton, D. (2017). Contextualising archaeological models with geological, airborne and terrestrial LiDAR data: The Ice Age landscape in Farndon Fields, Nottinghamshire, UK. Journal of Archaeological Science, 81, 31-48.

» Toumazet, J-P., Vautier, F., Roussel, E. y Dousteyssier, B. (2017). Automatic detection of complex archaeological grazing structures using airborne laser scanning data. Journal of Archaeological Science: Reports, 12, 569-579.

» Traviglia, A., Cowley, D. y Lambers, K. (2016). Finding Common Ground: Human and Computer Vision in Archaeological Prospection. AARGnews - The newsletter of the Aerial Archaeology Research Group, 53, 11-24.

» von Schwerin, J., Richards-Rissetto, H., Remondino, F., Spera, M. G., Auer, M., Billen, N., Loos, L., Stelson, L. y Reindel, M. (2016). Airborne LiDAR acquisition, post-processing and accuracy-checking for a 3D WebGIS of Copan, Honduras. Journal of Archaeological Science: Reports, 5, 85-104.

»Vosselman, G. y Maas, H. G. (2010). Airborne and terrestrial laser scanning. UK: Whittles $P$, CRC Press.

»Weiser, W. (1925-1926). Diario de Viaje y Fotografías de la VIII Expedición Arqueológica Benjamín Muniz Barreto a la Provincia de Catamarca. Archivo Documental de la División Arqueología del Museo de La Plata. Facultad de Ciencias Naturales y Museo, UNLP. Manuscrito inédito.

»Williams, V. y D’Altroy, T. N. (1998). El sur del Tawantinsuyu: un dominio selectivamente intensivo. Tawantinsuyu, 5, 170-178. 
"Williams, V., Villegas, M. P., Gheggi, M. S. y Chaparro, M. G. (2005). Hospitalidad e intercambio en los valles mesotermales del Noroeste argentino. Boletín de Arqueología PUCP, 9, 335-372.

»Zakšek, K., Oštir, K. y Kokalj, Z. (2011). Sky-view factor as a relief visualization technique. Remote Sens, 3, 398-415. 